\title{
Functional Recovery of Human Cells Harbouring the Mitochondrial DNA Mutation MERRF A8344G via Peptide-Mediated Mitochondrial Delivery
}

\author{
Jui-Chih Chang ${ }^{a}$ Ko-Hung Liu ${ }^{a} \quad$ Yu-Chi Lia Shou-Jen Kou ${ }^{b}$ Yau-Huei Weid, e \\ Chieh-Sen Chuang ${ }^{c}$ Mingli Hsieh ${ }^{f}$ Chin-San Liu ${ }^{a, c}$ \\ ${ }^{a}$ Vascular and Genomic Center, ${ }^{b}$ Department of Surgery, and ${ }^{c}$ Department of Neurology, Changhua \\ Christian Hospital, Changhua, ${ }^{d}$ Department of Biochemistry and Molecular Biology, School of Life Sciences, \\ National Yang-Ming University, ${ }^{e}$ Department of Medicine, Mackay Medical College, Taipei, and \\ fDepartment of Life Science, Tunghai University, Taichung, Taiwan, ROC
}

\section{Key Words \\ Cell-penetrating peptide $\cdot$ Mitochondrial delivery $\cdot$ Myoclonic epilepsy with ragged-red fibres syndrome • Mitochondrial functions - Mitochondrial biogenesis • Mitochondrial fusion/fission proteins}

\begin{abstract}
We explored the feasibility of mitochondrial therapy using the cell-penetrating peptide Pep-1 to transfer mitochondrial DNA (mtDNA) between cells and rescue a cybrid cell model of the mitochondrial disease myoclonic epilepsy with ragged-red fibres (MERRF) syndrome. Pep-1-conjugated wild-type mitochondria isolated from parent cybrid cells incorporating a mitochondria-specific tag were used as donors for mitochondrial delivery into MERRF cybrid cells (MitoB2) and mtDNA-depleted Rho-zero cells (Mito $\rho^{\circ}$ ). Forty-eight hours later, translocation of Pep-1-labelled mitochondria into the mitochondrial regions of MitoB2 and Mito $\rho^{\circ}$ host cells was observed (delivery efficiencies of 77.48 and $82.96 \%$, respectively). These internalized mitochondria were maintained for at least 15 days in both cell types and were accompanied by mitochondrial function recovery and cell survival by preventing mitochondria-dependent cell death. Mitochondrial homeostasis analyses showed that
\end{abstract}

peptide-mediated mitochondrial delivery (PMD) also increased mitochondrial biogenesis in both cell types, but through distinct regulatory pathways involving mitochondrial dynamics. Dramatic decreases in mitofusin-2 (MFN2) and dynamin-related protein 1/fission 1 were observed in MitoB2 cells, while Mito ${ }^{\circ}$ cells showed a significant increase in optic atrophy 1 and MFN2. These findings suggest that PMD can be used as a potential therapeutic intervention for mitochondrial disorders.

Copyright $\odot 2012$ S. Karger AG, Basel

\section{Introduction}

Mitochondria play a critical role in processes such as aerobic metabolism of glucose and fat, oxidative phosphorylation, calcium signalling, apoptosis and oxidative stress. Because all of these processes are important in determining the life span of eukaryotes, mitochondrial disorders are debilitating and often fatal. Unlike the nuclear genome, the mitochondrial genome is not protected by histones and is prone to high mutation rates and inefficient repair caused by extensive generation of oxygen radicals by redox reactions during aerobic respiration.

\begin{tabular}{ll}
\hline KARGER & $\begin{array}{l}\text { ○ 2012 S. Karger AG, Basel } \\
1424-862 \mathrm{X} / 13 / 0214-0160 \$ 38.00 / 0 \quad \text { Karger }\end{array}$ \\
$\begin{array}{l}\text { E-Mail karger@karger.com } \\
\text { www.karger.com/nsg }\end{array}$ & $\begin{array}{l}\text { This is an Open Access article licensed under the terms of the } \\
\text { Creative Commons Attribution-NonCommercial 3.0 Un- } \\
\text { ported license (CC BY-NC) (www.karger.com/OA-license), } \\
\text { applicable to the online version of the article only. Distribu- } \\
\text { tion permitted for non-commercial purposes only. }\end{array}$
\end{tabular}

Chin-San Liu, MD, PhD

Changhua Christian Hospital

Vascular and Genomic Center

135 Nanhsiao Street, Changhua 50094, Taiwan (ROC)

Tel. +886 4723 8595, ext. 4751, E-Mail $26602 @$ cch.org.tw 
Mitochondrial DNA (mtDNA) mutations that cause human mitochondrial diseases not only arise sporadically but may also be maternally inherited in different types of neurodegenerative diseases such as myoclonic epilepsy with ragged-red fibres (MERRF) syndrome. A mutation at the $A-G$ position of the tRNA ${ }^{\text {Lys }}$ gene in this disease is associated with severe defects in mitochondrial protein synthesis. This impairs electron transport chain assembly and respiratory chain activity, leading to cell death [1]. As with all mitochondrial disorders, there is no cure for MERRF, although mitochondrial therapies including coenzyme Q10, L-carnitine and various vitamins are commonly used to improve mitochondrial functions [2].

Accumulation of damaged mitochondria ( $>70-80 \%)$ that cannot be turned over through mitochondrial dynamics-triggered recovery of mitochondrial functions can also cause diseases [3]. This suggests that manipulating mitochondrial dynamics may provide a promising therapeutic strategy [4]. The mitochondrial dynamics network consists of continuous fusion and fission. The fusion/fission balance controls the fate of a depolarized mitochondrion, which is then either rescued by complementation or eliminated by autophagy. Mitochondrial fusion of premixed mutant and wild-type (WT) mtDNA before mitochondrial segregation during cell division buffers the effects of the mtDNA mutation and minimizes genetic drift of mutant mtDNA in daughter cells [3].

Mitochondrial transfer (mitotransfer) therapy, a variant of cytoplasmic transfer, is a method of gene therapy used for maternally inherited diseases [5]. Exogenous mitochondria are introduced by microinjection, and cellcell induction rapidly replaces the endogenous offending mtDNA [6, 7], restoring cell functions in vitro [8] and in vivo $[9,10]$, although the precise mechanism by which this occurs in host cells is unclear.

Based on this concept, we hypothesized that active induction of mitotransfer via a peptide delivery system could be a viable approach for mitochondrial therapy. Unlike most strategies that have limitations due to complex stability and cargo properties, cell-penetrating peptides appear to be very promising in increasing the bioavailability of compounds, such as drugs, and may play a critical role in treating certain diseases [11].

Pep-1 (KETWWETWWTEWSQPKKKRKV-cysteamine), a member of the cell-penetrating peptide family, is an amphipathic peptide with 3 domains, namely a hydrophobic tryptophan-rich motif, a hydrophilic lysinerich domain (KKKRKV) and a spacer domain (SQP). Pep-1 can efficiently deliver diverse fully biologically active peptides and proteins into cells via electrostatic and hydrophobic contacts with the cell membrane without requiring prior cross-linking or chemical modification. The mechanism of Pep-1 cell translocation is endosomal pathway-independent $[12,13]$ and does not affect the competitive binding of receptors, reporter genes, receptor internalization or intracellular calcium release in different cell lines [14], including mesenchymal stem cells [15], nor does it cause cytotoxicity in mammalian cells $[16,17]$. Although cell-penetrating peptides such as Pep-1 have been shown to efficiently improve the intracellular delivery of plasmid DNA, short interfering RNA, proteins, peptides and nanoparticles in vitro and in vivo [11, 18], their feasibility for delivery of organelles, such as mitochondria, remains unknown.

Here, we utilized a cybrid model of MERRF syndrome to demonstrate the feasibility of a therapeutic approach via Pep-1-mediated mitochondrial delivery (PMD) by examining the internalization of Pep-1-labelled mitochondria, recovery of mitochondrial functions, cell viability and mitochondrial homeostasis. Prior to isolation, WT mitochondria of donor cells were tagged with either MitoTracker dye or by genetic encoding of green fluorescent protein (GFP). Mitochondrial homeostasis was comprehensively evaluated by analysing mitochondrial biogenesis, including biogenetic gene expression, mtDNA copy numbers, mitochondrial contents and fusion-/fission-related dynamic proteins. Moreover, we analysed the differences between PMD-mediated regulation in different types of mitochondria-deficient cells, namely cybrid cells harbouring the $\mathrm{A} 8344 \mathrm{G}$ mutation in mtDNA and mtDNA-less $\left(\rho^{\circ}\right)$ cells, in order to clarify the role of mitochondria in this therapeutic mechanism and as an aid in the development of therapeutic strategies for the treatment of mitochondrial diseases.

\section{Materials and Methods}

Additional details are provided in the online supplementary materials (for all online supplementary material, see www. karger.com/doi/10.1159/000341981).

\section{Cell Culture and Culture Conditions}

The cybrid cell lines were kind gifts of Y.H. Wei [19]. Cybrids harbouring the A8344G mutation in mtDNA (MERRF B2 cybrid cells) at a rate of $85 \%$ were generated by fusing mtDNA-less human osteosarcoma $143 \mathrm{~B}$ cells (143B $\rho^{\circ}$ cells) with enucleated skin fibroblasts that were established from a patient with clinically proven MERRF syndrome according to the method described by King and Attardi [20]. Cybrid cells were grown in high-glucose DMEM (Gibco/Invitrogen, Carlsbad, Calif., USA) supplemented with $5 \%$ FBS (Gibco), $100 \mu \mathrm{g} / \mathrm{ml}$ sodium pyruvate (Gibco), $50 \mu \mathrm{g} /$ 
$\mathrm{ml}$ uridine (Sigma, St. Louis, Mo., USA) and 1\% penicillin-streptomycin (100 U/l penicillin G sodium, $100 \mathrm{mg} / \mathrm{l}$ streptomycin sulfate, Gibco) at $37^{\circ} \mathrm{C}$ in humidified $5 \% \mathrm{CO}_{2} / 95 \%$ air. Skin fibroblasts derived from a healthy subject and MERRF patients were cultured in low-glucose DMEM (Gibco) supplemented with 10\% FBS, $100 \mu \mathrm{g} / \mathrm{ml}$ sodium pyruvate and $1 \%$ penicillin-streptomycin at $37^{\circ} \mathrm{C}$ in humidified $5 \% \mathrm{CO}_{2} / 95 \%$ air.

\section{Mitochondrial Isolation and Delivery}

Mitochondria were isolated from parent 143B cells using a Mitochondria Isolation Kit for Cultured Cells according to the manufacturer's instructions (Pierce Chemical Co., Rockford, Ill., USA). Isolated mitochondria were quantified by determining protein concentrations using a bicinchoninic acid assay (Pierce), and the residual was ready to deliver immediately. The average amount of isolated mitochondria from $2 \times 10^{7}$ cells was $105 \pm$ $7.5 \mu \mathrm{g}$. Mitochondria were conjugated with Pep-1 (Anaspec, San Jose, Calif., USA) using a weight ratio of Pep-1 to mitochondria of $1,750: 1$ by incubation for $30 \mathrm{~min}$ at $37^{\circ} \mathrm{C}$ to form a complex. They were then transferred to $2 \times 10^{5}$ host cells grown on $10-\mathrm{cm}$ dishes with $10 \mathrm{ml}$ of regular medium for delivery for $48 \mathrm{~h}$. Prior to their isolation from donor cells, delivered mitochondria were tracked by tagging them with either mitochondrion-specific dyes or by genetic encoding of GFP. Prior to isolation, donor cells of mitochondria were labelled with a red fluorescent mitochondrial dye whose uptake was dependent on membrane potential (100 nM final concentration; MitoTracker Red CMXRos, Invitrogen-Molecular Probes, Eugene, Oreg., USA) for $20 \mathrm{~min}$ in a $\mathrm{CO}_{2}$ incubator. By determining the fluorescence intensity before and after isolation, we found that only $33.3 \pm 2.94 \%$ of mitochondria (105 $\pm 4.04 \mu \mathrm{g}$ of mitochondrial protein) could be separated from donor cells if light bleaching during the isolation process was not considered. Delivery efficiency of dye-labelled mitochondria was assessed using a Cytomics FC 500 flow cytometer (Beckman Coulter Inc., Fullerton, Calif., USA). For further verification of mitochondrial internalization over the long term, GFP expression in host cells after delivery of GFP-tagged mitochondria was analysed by Western blot at different time points.

\section{Plasmids and Transfection}

For long-term tracking of internalized Pep-1-labelled mitochondria (Pep-1-Mito) in treated cells, mitochondria that were to be delivered were genetically encoded with GFP prior to isolation. For transient transfection, mitochondrial donor cells $(80 \%$ confluent) were transfected using a PureFection transfection reagent (System Biosciences, Mountain View, Calif., USA) with plasmid DNA of pAC-GFP-mito (Clontech, Palo Alto, Calif., USA) at a $1.5: 1$ ratio of reagent $(60 \mu \mathrm{l})$ to DNA $(40 \mu \mathrm{g})$ as per the manufacturer's instructions. After $36 \mathrm{~h}$, the transfected cells were placed in a normal growth medium for $4 \mathrm{~h}$ and treated with G418 (0.6 $\mathrm{mg} / \mathrm{ml}$; Sigma-Aldrich, St. Louis, Mo., USA) for clonal selection.

\section{DNA Extraction and PCR-Restriction Fragment Length}

Polymorphism Analysis

Total DNA was extracted from cells with or without mitochondrial delivery for 2 days using a Gentra Puregene Cell Kit (Qiagen GmbH, Hilden, Germany). To detect the A8344G mutation, a segment of mtDNA was amplified by PCR (MJ Research PTC2000 Peltier ThermoCycler, Biorad, Hertfordshire, UK). After the last cycle, the products were labelled with $0.3 \mu$ l of fluores- cence-labelled nucleotide (FdNTP) (Perkin-Elmer, Foster City, Calif., USA) and digested with NaeI. A 223-bp PCR product was cleaved by NaeI into 197 - and 26-bp fragments. The digested DNA mixture was further analysed by $4 \%$ agarose gel electrophoresis.

\section{Confocal Microscopy}

After PMD for 2 days, the internalization of Pep-1-labelled mitochondria was assessed by cell membrane staining using an amphipathic molecule $(5 \mu \mathrm{g} / \mathrm{ml})$ that provided a lipophilic moiety for membrane loading (CellMask ${ }^{\mathrm{TM}}$ Deep Red cytoplasmic/nuclear stain, Invitrogen-Molecular Probes). Cell nuclei were stained with Hoechst 33342 (Invitrogen-Molecular Probes) before microscopic analysis. To further confirm the location and interaction with endogenous mitochondria in host cells, mitochondria in the rescued cells were labelled by adding a green fluorescent mitochondrial dye to the cultures (100 nM final concentration; MitoTracker Green, Invitrogen-Molecular Probes) for $20 \mathrm{~min}$ in a $\mathrm{CO}_{2}$ incubator. This dye was concentrated in active mitochondria by a process that was independent of the mitochondrial membrane potential. Mitochondrial morphology was observed with an inverted LSM510 Laser Scanning Microscope (Axiovert 100, Carl Zeiss, Göttingen, Germany) with a configuration appropriate for detecting the maximum signals from mitochondria-labelled cells. To observe the locations of delivered mitochondria labelled with MitoTracker Red CMXRos, a three-dimensional section was analysed. To characterize the distribution of delivered mitochondria around host mitochondria, integration of line scans through the $\mathrm{z}$-axis using Zeiss LSM510 software provided a longitudinal view of the synaptic site.

\section{Morphological Identification of Mitochondria}

Mitochondrial morphology in individual cells was evaluated by fluorescence microscopy. Fragmented mitochondria were shortened, punctate and sometimes rounded, whereas filamentous mitochondria exhibited thread-like tubular structures. The mitochondria within one cell were often either filamentous or fragmented. In rare cases of mixed morphologies, we classified those cells with obvious morphological fragmentation based on the majority $(>70 \%)$ of fragmented mitochondria (fragment size was identified as 3-5 $\mu \mathrm{m}$ ) according to a previous study [21]. The percentage of cells with mitochondrial elongation was determined by subtracting the proportion of cells with fragmentation from the total cells.

\section{Mitochondrial Function Assays}

Mitochondrial functional assessments were made by comprehensive evaluations that included a mitochondrial membrane potential assay, oxygen consumption, intracellular ATP concentration and lactate production rate. Detailed descriptions are provided in the supporting online supplementary methods. Changes in mitochondrial membrane potential were determined by flow cytometry using the fluorescent dyes rhodamine 123 (Sigma-Aldrich) and JC1 (Invitrogen-Molecular Probes). By comparisons with cells with rotenone (100 $\mu \mathrm{M}$; Sigma-Aldrich)induced respiratory depression, the mitochondrial uptake of rhodamine 123 was analysed to reflect membrane potential function. A population of cells with high levels of JC1 monomers accompanied by low levels of JC1 aggregates represented a population with depolarized mitochondrial membrane potential. We measured dissolved oxygen during the bioprocess of rescuing 
cells using a NeoFox Oxygen sensor (Ocean Optics, Dunedin, Fla., USA). These data provided the net oxygen consumption rate (percentage difference between before and after glucose-induced values for $1 \mathrm{~h}$ ) normalized to the number of available cells (percentage of oxygen consumed per minute per $10^{6}$ cells). Intracellular ATP levels were measured with Bioluminescent Somatic Cell Assay Kits (Sigma-Aldrich) according to the manual. Luminescence intensity was determined with a FLUOstar OPTIMA multidetection microplate reader (BMG Labtech, Offenburg, Germany). ATP levels were then determined from a standard curve of ATP dilutions and given as picomoles per $10^{6}$ cells after normalizing to the cell number. The lactate production rate was determined with a Lactate Reagent kit (Trinity Biotech Plc., Bray, Ireland). The concentration of extracellular lactate was determined using a standard curve of lactate dilutions and with results normalized to the total cell number and divided by the time of incubation.

\section{Calcein-AM/Propidium Iodide Double Staining}

Cell viability was assessed by the retention of acetomethoxy derivate of calcein (calcein-AM) (Invitrogen Corporation, Carlsbad, Calif., USA) and propidium iodide (PI; Invitrogen) uptake using a Nikon TE 300 inverted fluorescence microscope (Nikon, Tokyo, Japan). Cells were seeded in 24 -well plates $\left(8 \times 10^{3}\right.$ cells/ well) and cultured with $2 \mathrm{ml}$ of the same medium without glucose for 4 days without changing the medium. Cultured cells were rinsed carefully with PBS and incubated with $1 \mu \mathrm{g} / \mathrm{ml}$ calcein$\mathrm{AM}$ and $10 \mu \mathrm{g} / \mathrm{ml} \mathrm{PI}$ in PBS at $37^{\circ} \mathrm{C}$ for $35 \mathrm{~min}$. Images were acquired using a Princeton Instruments MicroMax CCD camera. Cell viability was expressed as the ratio of calcein-positive cells to the sum of calcein-positive and PI-positive cells.

\section{Western Blot Analysis}

Aliquots of whole-cell lysates with $25 \mu \mathrm{g}$ of protein were fractionated, separated electrophoretically on a $12 \%$ SDS-polyacrylamide gel (Bio-Rad Laboratories, Richmond, Calif., USA) and blotted onto a piece of polyvinylidene difluoride membrane (Amersham Biosciences, Buckinghamshire, UK). Non-specific binding was blocked with $5 \%$ skim milk in Tris-buffered saline with Tween 20 (Sigma-Aldrich). The membrane was blotted with primary antibodies that included cytochrome $c$ (cyto $c$; 1:100 dilution; BD Biosciences, Pharmingen, San Diego, Calif., USA), procaspase-3 (1:100 dilution; Santa Cruz Biotechnology, Calif., USA), optic atrophy type 1 (OPA1; 1:500 dilution; BD Biosciences, Pharmingen), mitofusin-2 (MFN2; 1:500 dilution; Sigma-Aldrich), mitochondrial fission 1 (Fis1; 1:500 dilution; Axxora, San Diego, Calif., USA) and $\beta$-actin (1:1,000 dilution; Chemicon, Temecula, Calif., USA). After incubation with a horseradish peroxidase-conjugated secondary antibody, protein intensity was determined using an enhanced chemiluminescence reagent (Immobilon Western, Millipore, Billerica, Calif., USA).

\section{Active Caspase-3/-7 Activity}

Quantification of caspase-3/-7 activation was assessed using a fluorochrome-labelled inhibitor of caspase (FLICA) apoptosis detection kit (Immunochemistry Technologies, Bloomington, Minn., USA) according to the manufacturer's protocol. A $30 \times$ FLICA solution $(10 \mu \mathrm{l})$ was added to cells (approx. $1 \times 10^{6} / 300 \mu \mathrm{l}$ culture medium), incubated at $37^{\circ} \mathrm{C}$ in $5 \% \mathrm{CO}_{2}$ for $1 \mathrm{~h}$, washed twice and resuspended in wash buffer. Caspase-3/-7 activity was determined by flow cytometry as the ratio of fluorescence-positive cells to unstained cells. For each sample, $1 \times 10^{4}$ cells were analysed using WinMDI 2.9 software.

\section{RNA Extraction and Quantitative Real-Time RT-PCR Analysis}

Total RNA was isolated from cells using TRIzol reagent (Invitrogen). Total RNA was further purified with a NucleoSpin ${ }^{\circledR}$ RNA II Kit (Macherey-Nagel, Düren, Germany) and reverse transcribed using a Transcriptor First Strand cDNA Synthesis kit (Roche Applied Science, Indianapolis, Ind., USA). mRNA expressions were quantitatively determined by real-time RT-PCR using SYBR Green PCR Master Mix (Roche Applied Science) and an ABI Prism 7300 system (Applied Biosystems, Foster City, Calif., USA). mRNA expressions were normalized to $\beta$-actin mRNA and given as relative expression levels.

\section{mtDNA Copy Numbers}

An aliquot of $50 \mathrm{ng}$ of DNA was subjected to quantitative PCR using a LightCycler-FastStart DNA Master SYBR Green I kit (Roche Applied Science). DNA fragments of the nicotinamide adenine dinucleotide dehydrogenase subunit 1 (ND1) gene (mtDNA-encoded) and the $\beta$-actin gene (nuclear DNA-encoded, used as an internal control) were amplified with specific primer pairs. The relative mtDNA copy number was determined by normalizing the crossing points on quantitative PCR curves between the ND1 and $\beta$-actin genes using RelQuant software (Roche Applied Science).

\section{Mitochondrial Mass Assay}

Cells were incubated in fresh medium with $2.5 \mu \mathrm{M}$ nonyl acridine orange (Molecular Probes) for $10 \mathrm{~min}$ at $37^{\circ} \mathrm{C}$ in the dark and harvested in HEPES buffer ( $\mathrm{pH}$ 7.4). The fluorescence intensity of $1 \times 10^{4}$ cells was recorded using a flow cytometer with an excitation wavelength of $488 \mathrm{~nm}$ and emission wavelength of 535 nm.

\section{Mitochondrial Calcium Uptake}

The mitochondrial $\mathrm{Ca}^{2+}\left(\left[\mathrm{Ca}^{2+}\right]_{\text {mito }}\right)$ level was quantified after loading cells with the $\mathrm{Ca}^{2+}$ fluorophore rhod-2 (Molecular Probes, Eugene, Oreg., USA), a selective fluorescent indicator for $\left[\mathrm{Ca}^{2+}\right]_{\text {mito }}$. For confocal fluorescent images of double-labelled cells, cybrid cells were pre-incubated for 10 min in Hank's Buffered Salt Solution (HBSS) at $37^{\circ} \mathrm{C}$ with $100 \mathrm{nM}$ MitoTracker Green (Molecular Probes). The 2-step cold loading/warm incubation protocol provides exclusive loading of rhod-2 into mitochondria [22]. Rhod-2 fluorescence was excited at $560 \mathrm{~nm}$, and emitted fluorescence was collected through a 590-nm-long pass barrier filter. The fluorescence intensity of rhod-2 was quantified using flow cytometry. To confirm $\left[\mathrm{Ca}^{2+}\right]_{\text {mito }}$, the fluorescence intensity of rhod- 2 was determined again in cells after an additional warm incubation for $40 \mathrm{~min}$ in $0.15 \%$ hydrogen peroxide $\left(\mathrm{H}_{2} \mathrm{O}_{2}\right)$.

\section{Statistical Analysis}

All experiments were performed with triplicate samples and were repeated at least 3 times. Results are given as means \pm SD. Comparisons of two experimental conditions were made by a paired Student's t test. A p value of $<0.05$ was considered to be statistically significant. 


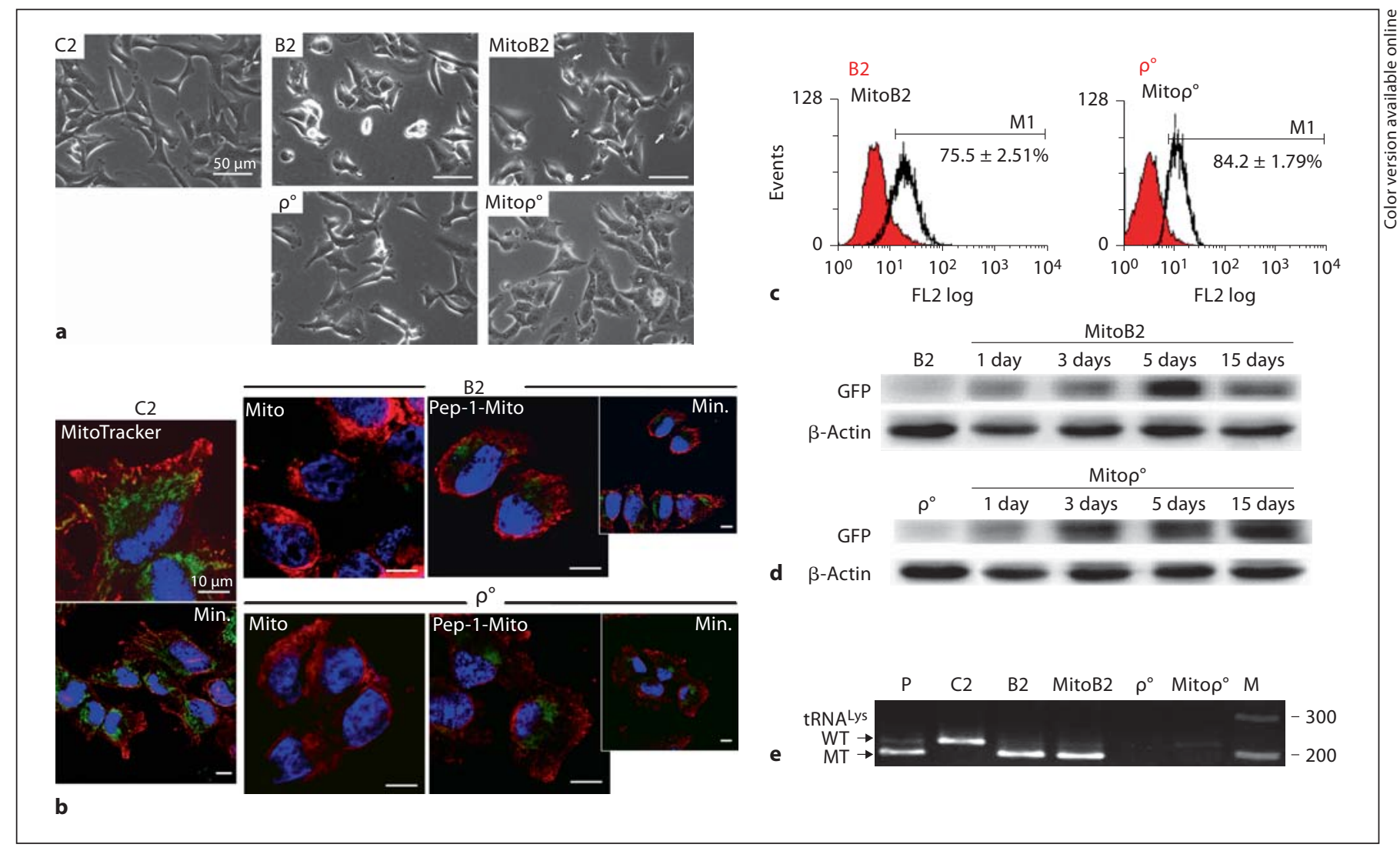

Fig. 1. Morphology and internalization of Pep-1-Mito in treated MERRF cybrid and $\rho^{\circ}$ cells. a Comparison of morphological differences before and after 1 day of treatment with Pep-1-Mito in MERRF cybrid (MitoB2) and $\rho^{\circ}\left(\right.$ Mito $\left.^{\circ}\right)$ cells. As indicated by the arrows, a marked change in morphology with spreading out was only observed in MitoB2 cells. b After staining cells with CellMask Deep Red plasma membrane (red) and nuclear (Hoechst 33348 , blue) stains, internalization of mitochondria preloaded with MitoTracker Green dye (green) prior to isolation with Pep-1 conjugation (Pep-1-Mito) or not (mitochondria alone, Mito) was observed in treated cells by confocal microscopy at different magnifications after 2 days of treatment. The inherent mitochondria of parent cybrid cells were co-stained with MitoTracker Green dye as a positive control (C2). Min. = Minimized view. $\mathbf{c}$ Internalization efficiency was assessed by fluorescence intensity using flow cytometry for MERRF cybrid (B2) and $\rho^{\circ}$ cells before and after

\section{Results}

\section{Cell Morphology and Internalization of}

Pep-1-Labelled Mitochondria

MERRF cybrid cells (B2) had a more rounded morphology compared with parent cybrid 143B cells (C2; fig. 1a). However, MERRF cybrid cells (MitoB2) with overnight PMD treatment exhibited normal spread-out treatment with Pep-1-Mito preloaded with MitoTracker Red dye prior to isolation (MitoB2 and Mito $\rho^{\circ}$ ). $\mathbf{d}$ Delivery of mitochondria that were genetically targeted with GFP was used to track the internalization process in cells during long-term culture. GFP expression in MERRF cybrid (B2) and $\rho^{\circ}$ cells before and after treatment with Pep-1-Mito (MitoB2 and Mito $\rho^{\circ}$ ) was individually examined by Western blotting at different time points. $\beta$-Actin was used as an internal control for judgment of protein contents. e PCR-restriction fragment length polymorphism genotyping of point mutations (A8344G tRNA ${ }^{\text {Lys }}$ ) in MERRF cybrid and $\rho^{\circ}$ cells was performed before and after 1 day of treatment with Pep-1Mito. Comparisons of MERRF patient-derived fibroblasts harbouring $>85 \%$ of the A8344G mutation (positive control, $\mathrm{P}$ ) with the proportions of WT and A8344G tRNA ${ }^{\text {Lys }}$ mutation (MT) were made by separating DNA fragments with polyacrylamide gel electrophoresis. $\mathrm{M}=$ DNA marker.

morphologies (fig. 1a, arrows) relative to non-treated cells (B2). In contrast, this phenomenon was not remarkable in mtDNA-depleted $\rho^{\circ}$ cells after treatment (Mito $\rho^{\circ}$; fig. 1a).

Initially, mitochondrial internalization was demonstrated by the presence of a tag (dye or genetic modification with GFP) in treated host cells (fig. 1b-d). Compared with $\mathrm{C} 2$ cells stained separately with markers for either 
mitochondria (MitoTracker, green), cell membranes (red) or nuclei (blue), green fluorescence was only observed in the cytoplasm of MERRF cybrid cells and $\rho^{\circ}$ cells treated with Pep-1-Mito, but not in cells treated with mitochondria alone (fig. 1b).

Flow cytometry analysis revealed that $75.5 \pm 2.51 \%$ and $84.2 \pm 1.79 \%$ of treated MERRF cybrid (MitoB2) and $\rho^{\circ}\left(\mathrm{Mito}^{\circ}\right)$ cells, respectively, had marked fluorescence derived from internalized Pep-1-Mito preloaded with MitoTracker Red CMXRos (fig. 1c). Pep-1-Mito internalization was confirmed by time course tracking of GFP expression in Pep-1-Mito-GFP-tagged treated cells. Both MitoB2 and Mito $\rho^{\circ}$ cells exhibited GFP expression 1 day after treatment, which increased significantly during the following days compared to each untreated group (fig. 1d). GFP expression remained for at least 15 days after treatment in both groups, although there was a decrease in GFP expression in the MitoB2 group at the 15th day of treatment (fig. 1d).

To determine the viability of PMD as a treatment strategy, the mtDNA tRNA ${ }^{\text {Lys }}$ A8344G polymorphism in rescued cells was analysed by PCR-restriction fragment length polymorphism 2 days after treatment. An increased proportion of WT mtDNA was observed in both the MitoB2 and Mito $\rho^{\circ}$ cells compared with untreated cells (fig. 1e), whereas the proportion of mutant tRNA ${ }^{\text {Lys }}$ A8344G mtDNA was not affected in the MitoB2 group (fig. 1e) compared to the untreated group (B2).

\section{Mitochondrial Distribution and Morphology after \\ Pep-1 Delivery}

To further examine the localization of the internalized mitochondria, the total mitochondria in treated cells were labelled with MitoTracker Green dye after 2 days of delivery. The confocal images in figure 2 show the interactions of Pep-1-Mito and endogenous mitochondria in treated MERRF cybrid cells (MitoB2 and Mito ${ }^{\circ}$; fig. 2a, b). As indicated by the arrows in figure 2a, Pep-1-Mito (red) appeared to aggregate as granules around the host mitochondria (green) in MitoB2 and Mito $\rho^{\circ}$ cells. Threedimensional reconstructions of the confocal images confirmed the co-localization of Pep-1-Mito and host mitochondria in treated cells (fig. $2 \mathrm{~b}$ ), suggesting that once internalized, exogenous mitochondria came in direct contact with the host mitochondria.

Localization of exogenous mitochondria following internalization was also examined in primary skin fibroblasts in which mitochondrial morphology and distribution are more readily visible (fig. 2c) compared to the cybrid cell models (fig. 2a). Primary skin fibroblasts from healthy individuals or MERRF patients were generated at the same time. Excessively fragmented mitochondria with shortened, punctate and sometimes rounded morphologies were observed in MERRF fibroblasts, whereas filamentous mitochondria with a thread-like tubular structure were found in normal fibroblasts (fig. 2c).

Internalization of Pep-1-Mito in MERRF fibroblasts was verified by the presence of fluorescence-positive expression (red) in cells treated with Pep-1-Mito and in MERRF cybrid cells, but not in cells treated with mitochondria alone (fig. 2c, 1b). The three-dimensional reconstructions of confocal images again revealed that the internalized mitochondria had co-localized with endogenous mitochondria in treated cells (fig. 2d). Quantification of mitochondrial morphology after 2 days of MitoPep-1 treatment in MERRF fibroblasts revealed a significant increase in the percentage of cells with elongated morphologies from $27 \pm 2.5 \%$ (control group) to $62 \pm$ $4.9 \%$ (fig. $2 \mathrm{c} ; \mathrm{p}<0.05$ ). This was in contrast to the results of treatment with mitochondria alone in MERRF fibroblasts (32 $\pm 7.5 \%$ ), for which there was no significant difference relative to untreated cells $(27 \pm 2.5 \%$, control group; fig. 2c).

\section{Recovery of Mitochondrial Functions and Cell}

Viability in Rescued Cells

Mitochondrial membrane potential was determined using the fluorescent dyes rhodamine 123 and JC1 after delivery for 2 days. Parent cybrid cells (C2) with rotenone $(100 \mu \mathrm{M})$-induced mitochondrial swelling were used as a positive control to assess the recovery of mitochondrial functions with rhodamine 123 staining. Relative to $\mathrm{C} 2$ cells, a significant decrease in rhodamine 123 fluorescence intensity was observed in MERRF cybrid and $\rho^{\circ}$ cells as well as in rotenone-treated cells (fig. 3a; p < 0.05). PMD significantly increased rhodamine 123 fluorescence intensity in treated cells $(\mathrm{p}<0.05)$, which reflected approximately 1.3- and 1.6-fold increases in MitoB2 and Mito $\rho^{\circ}$ cells, respectively, compared to each untreated group (B2 and $\rho^{\circ}$ ). Moreover, these results consistently showed that the population of cells with mitochondrial membrane potential depolarization $(\Delta \psi \mathrm{m})$ also had significantly decreased JC1 staining by approximately 4.4and 16.7-fold in MitoB2 and Mito $\rho^{\circ}$ cells, respectively (fig. 3b; $\mathrm{p}<0.05$ ).

In addition to the changes in mitochondrial membrane potential, PMD also induced changes in oxygen consumption and in intracellular ATP and extracellular lactate production. Increases of 1.7- and 2.1-fold in oxygen consumption relative to each untreated group were 


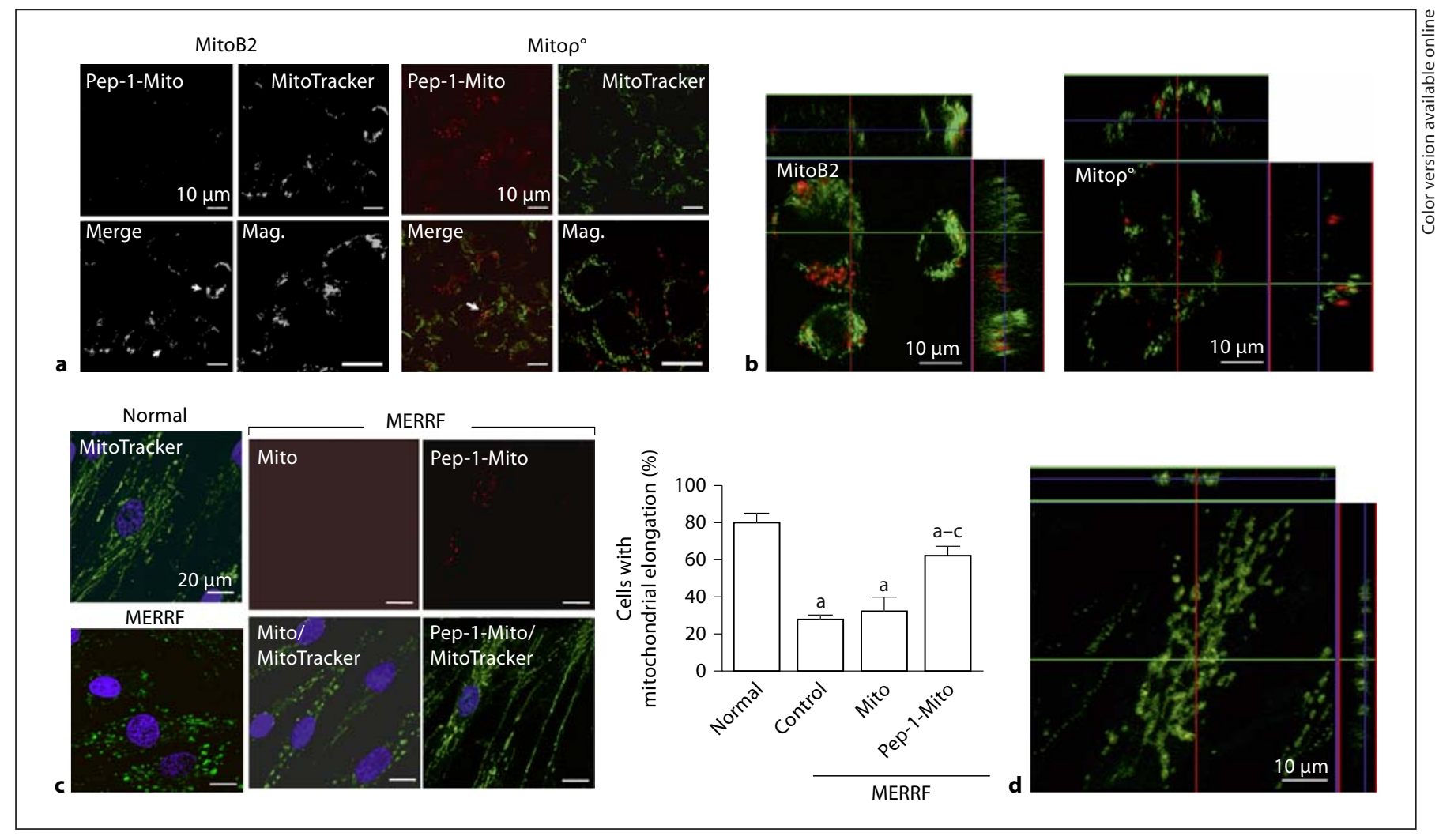

Fig. 2. Location and spatial relationships of internalized mitochondria with endogenous mitochondria in treated cells. After 2 days of treatment with Pep-1-Mito preloaded with MitoTracker Red dye, the total mitochondria in host cells were stained with MitoTracker Green dye to observe the interactions of internalized mitochondria (red) with endogenous mitochondria (green) in treated MERRF cybrid and $\rho^{\circ}$ cells (MitoB2 and Mito $\rho^{\circ} ; \mathbf{a}, \mathbf{b}$ ) and in normal and MERRF fibroblasts $(\mathbf{c}, \mathbf{d})$ by confocal microscopy. As indicated by arrows in a, Pep-1-Mito preferentially aggregated around host mitochondria. Three-dimensional voxel reconstructions of confocal images were used to analyse the colocalization relationships between internalized Pep-1-Mito (red) and endogenous mitochondria (green) in treated cells (b, d). Mitochondrial internalization in treated MERRF fibroblasts was confirmed by comparing with treatment with mitochondria alone without Pep-1 conjugation (Mito; c). Quantification of mitochondrial morphology was performed by determining the percentages of elongated mitochondria in MERRF fibroblasts before (control, Con) and after 2 days of treatment with mitochondria alone (Mito) and Pep-1-Mito (c). ${ }^{\mathrm{a}} \mathrm{p}<0.05$ : significant difference compared with the normal group; ${ }^{b} \mathrm{p}<0.05$ : significant difference compared with the control group; ${ }^{\mathrm{c}} \mathrm{p}<0.05$ : significant difference compared with the mitochondria alone group $(n=4)$. Mag. = Magnified view . observed in MitoB2 and Mito $\rho^{\circ}$ cells, respectively (fig. 3c), which reflected an efficient electron transport chain. Rescued cells exhibited significant induction of ATP synthesis (fig. 3d) accompanied by a marked reduction in lactate production after 2 days of delivery (fig. 3e; $p<$ 0.05 ), which was sustained until the 8 th day after delivery (fig. 3d, e). These results consistently indicated that rescued cells regained mitochondrial function, which was similar to that in the parent cybrid cells.

Mitochondrial dysfunction induces premature cell death once external energy support is removed. The mitochondrial dysfunctional MERRF cybrid and $\rho^{\circ}$ cells began to round up, became detached from the surface and died under glucose starvation when cultured for 4 days without any medium exchange (fig. 4a, b). This phenomenon was not detected in either the rescued cell groups (MitoB2 and Mito $\rho^{\circ}$ ) or the parent cybrid cells (C2; fig. 4a). This was confirmed with cell viability assays when green fluorescence was expressed again (calceinAM-labelled live cells), accompanied by a decrease in red fluorescence (PI-labelled dead cells) in treated MitoB2 and Mito $\rho^{\circ}$ cells (fig. $4 \mathrm{~b}$ ) as compared to each untreated group. Quantification of cell viability showed 1.73- and 2.72-fold increases in MitoB2 and Mito $\rho^{\circ}$ cells, respectively, which were equivalent to that of the control group (fig. 4b, c). 


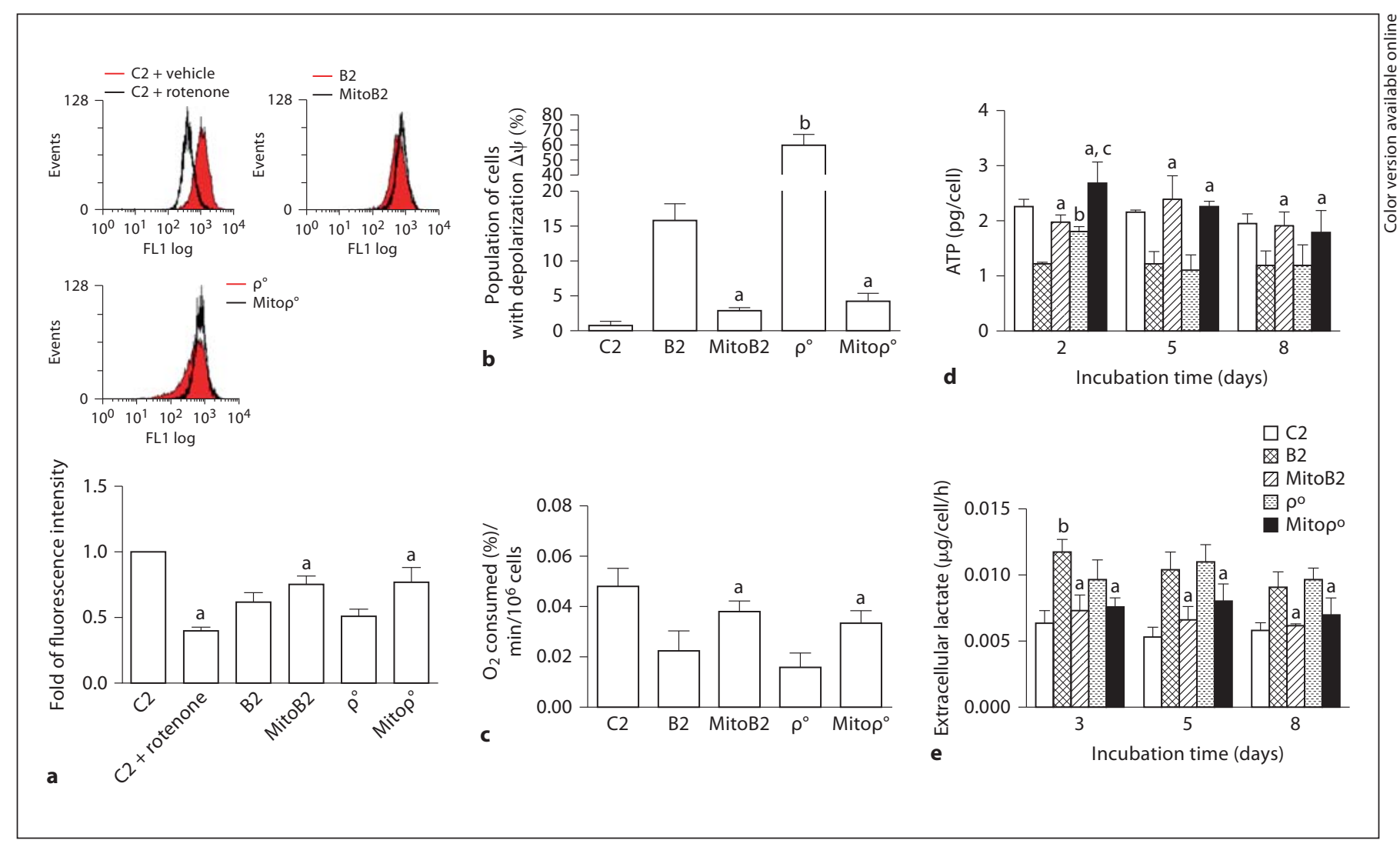

Fig. 3. Comprehensive analysis of mitochondria functional recovery in cells treated with Pep-1-Mito for 2 days. a, b Mitochondrial membrane potential was assessed by staining with rhodamine 123 (a) and JC1 (b). Rotenone-induced mitochondrial depolarization in parent cybrid cells (C2) was used to assess rhodamine 123 fluorescence by flow cytometry in treated cells and MERRF cybrid and $\rho^{\circ}$ cells with Pep-1-Mito treatment (MitoB2 and Mito $\rho^{\circ}$ ) or without treatment (B2 and $\rho^{\circ}$; a). The percentage of cells with high amounts of JC1 monomers accompanied by low amounts of JC1 aggregates reflected the level of mitochondrial

Mitochondria-mediated cell death was also analysed using cyto $c$ and caspase-3/-7 activation in total cell lysates. After PMD for 2 days, expression of cyto $c$ and procaspase-3 was significantly decreased in rescued cells (fig. $4 \mathrm{~d}$, e; $\mathrm{p}<0.05$ ). Caspase-3/-7 activation, as determined by calculating the percentage of FLICA-positive cells, confirmed the downregulation of procaspase-3/-7 activity in both rescued cell types (1.4- and 2.1-fold decreases in MitoB2 and Mito $\rho^{\circ}$ cells, respectively) after PMD for 2 days (fig. 4f). Taken together, these results demonstrate that PMD not only restores mitochondrial function but also reduces the susceptibility to cell death caused by mitochondrial dysfunction. depolarization (b). c Oxygen consumption in treated cells was analysed with an oxygen sensor. d, e The time course of cellular ATP synthesis (d) and extracellular lactate production (e) was determined with a bioluminescent somatic cell assay kit and a lactate reagent kit for cells with and without Pep-1-Mito treatment at different time points. ${ }^{a} \mathrm{p}<0.05$ : significant difference compared with the $\mathrm{C} 2$ group or mock control group (B2 or $\rho^{\circ}$ ); ${ }^{\mathrm{b}} \mathrm{p}<0.05$ : significant difference between B2 and $\rho^{\circ}$ groups; ${ }^{\mathrm{c}} \mathrm{p}<$ 0.05: significant difference between MitoB2 and Mito $\rho^{\circ}$ groups $(\mathrm{n}=3)$.

\section{Mitochondrial Biogenesis, Contents and Dynamic Proteins in Rescued Cells}

Regulation of mitochondrial homeostasis was determined by comprehensive analysis of mitochondrial biogenesis, content and dynamic proteins. PMD for 2 days significantly increased the gene expression of biogenesisrelated nuclear respiratory factor 1 (NRF-1) and mitochondrial transcription factor A (Tfam) in rescued cells relative to untreated cells (1.53- and 18.9-fold increases, respectively, in MitoB2 cells, and 2.16- and 4.78-fold increases, respectively, in Mito $\rho^{\circ}$ cells; $\left.\mathrm{p}<0.05\right)$. In contrast, the expression of peroxisome proliferator-activated receptor $\gamma$ coactivator- $1 \alpha$ gene in treated MERRF cybrid 


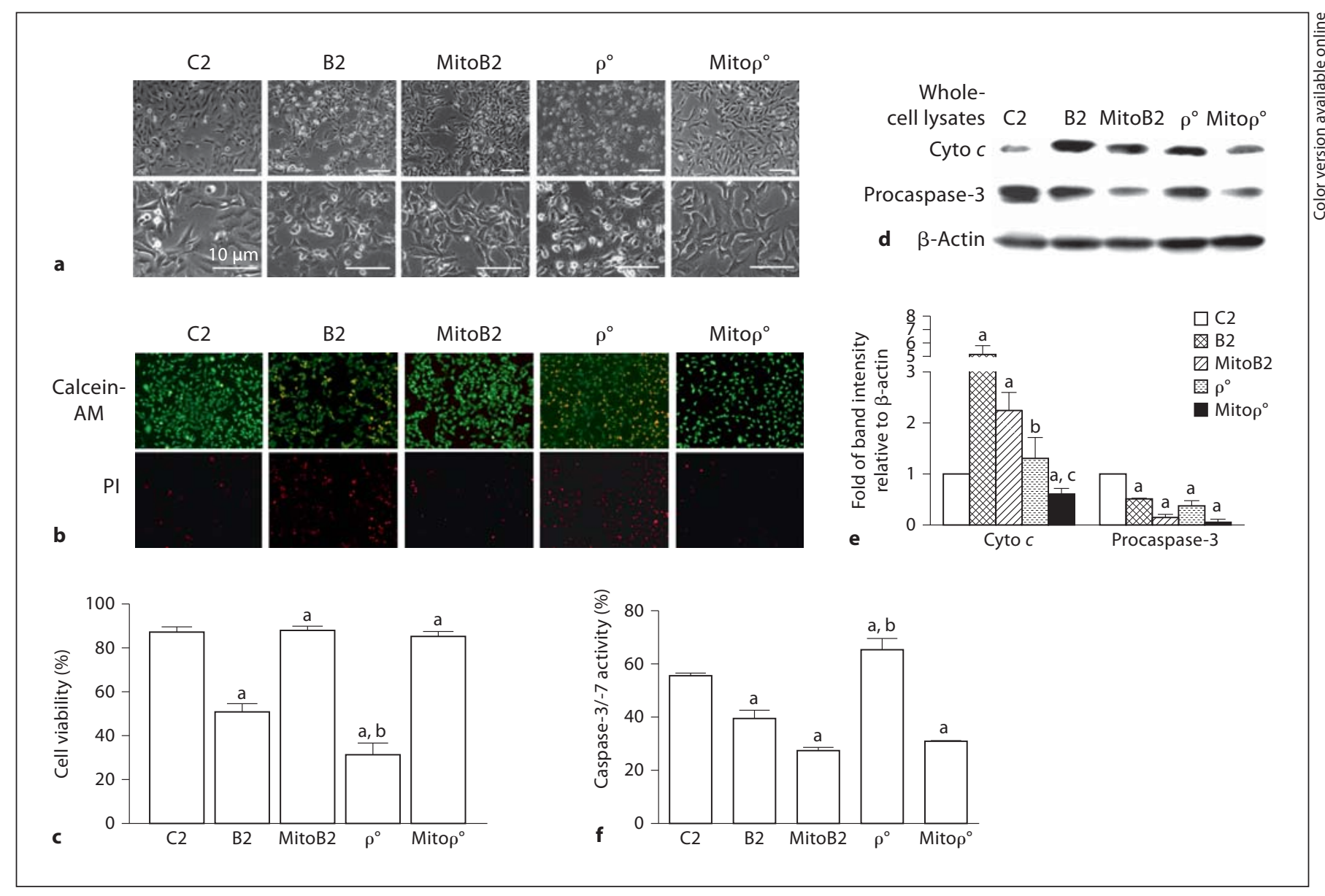

Fig. 4. Cell viability after mitochondrial delivery. a-c Cell morphology (a), live/dead cell (calcein-AM/PI) double staining (b) and quantification of cell viability (minus the percentage of PIpositive cells; c) were assessed in MERRF cybrid (B2) and $\rho^{\circ}$ cells either with Pep-1-Mito treatment (MitoB2 and Mito $\rho^{\circ}$ ) or not after 4 days of glucose starvation. d, e After 2 days of mitochondrial delivery, protein expression of cyto $c$ and procaspase- 3 in whole-cell lysates was analysed (d) and quantified by Western blotting (e). f Caspase-3/-7 activity was determined by carboxyfluorescein FLICA analysis using flow cytometry. Parent cybrid cells (C2) without mitochondrial delivery were used as the control. ${ }^{a} \mathrm{p}<0.05$ : significant difference compared with the $\mathrm{C} 2$ group or mock control group (B2 and $\rho^{\circ}$ ); ${ }^{\mathrm{b}} \mathrm{p}<0.05$ : significant difference between B2 and $\rho^{\circ}$ groups; ${ }^{c} \mathrm{p}<0.05$ : significant difference between MitoB2 and Mito $\rho^{\circ}$ groups $(n=3)$.

1.15 -fold relative to untreated cells at the 5 th day after delivery (fig. 5c). This was contrary to the results observed in treated $\rho^{\circ}$ cells, which showed a significant reduction in mitomass, which was even lower than that in parent cybrid cells (fig. 5d; p < 0.05). These results revealed that PMD restored mitochondrial function via the upregulation of mitochondrial biogenesis.

Based on the distinct mitomass expressions, mitochondria-dynamic proteins that allow for redistribution of mitochondrial components were examined, including both fusion (OPA1, MFN2) and fission proteins [fission 1 protein (Fis1), dynamin-related protein 1 (Drp1)]. Two 


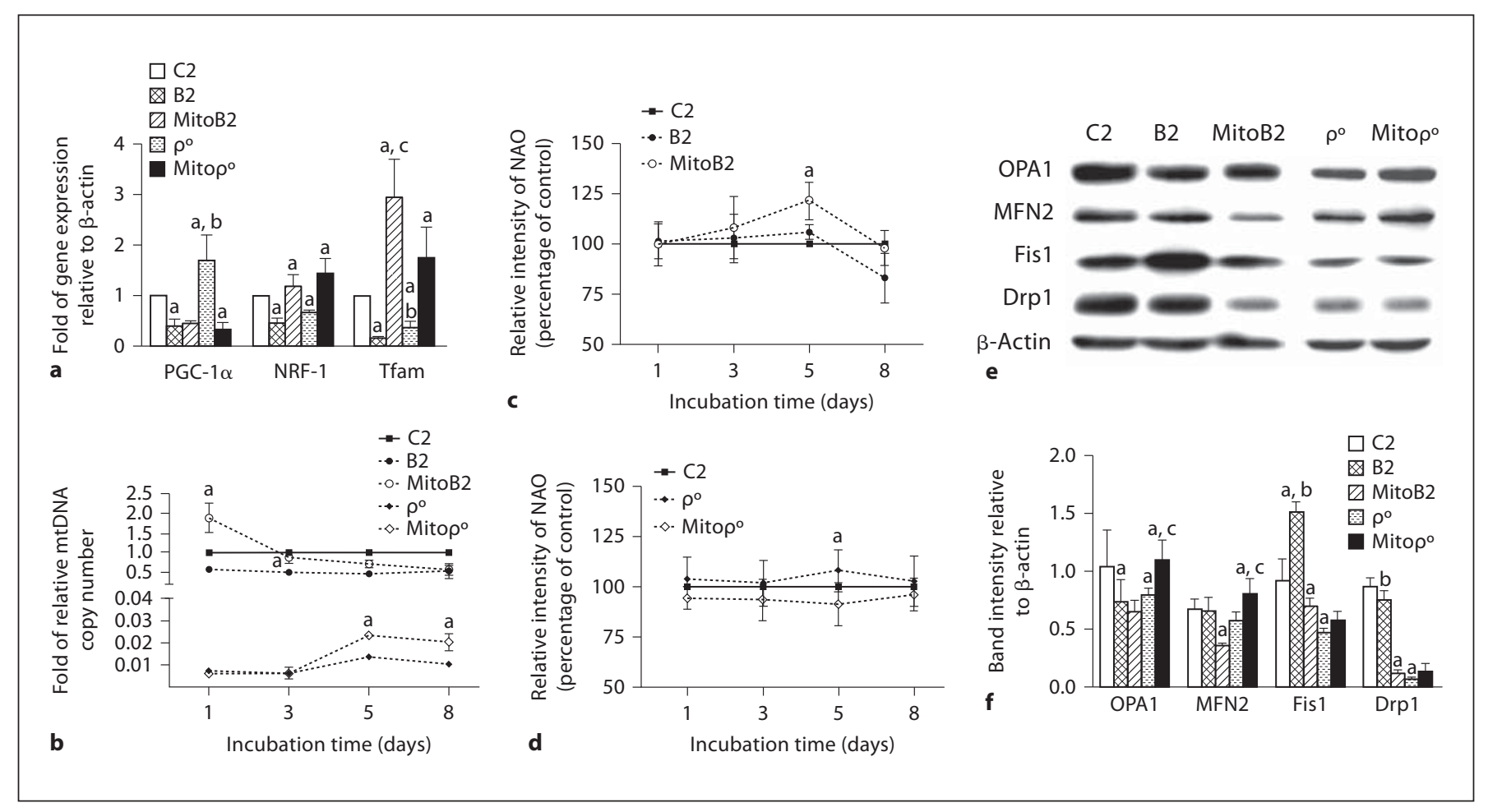

Fig. 5. Comprehensive assessment of mitochondrial homeostasis including mitochondrial biogenesis, mitochondrial content and dynamic proteins in cells after mitochondrial delivery. a Mitochondrial biogenesis-related genes PGC-1 $\alpha$, NRF-1 and Tfam were analysed by real-time RT-PCR in MERRF cybrid (B2) and $\rho^{\circ}$ cells after 2 days of Pep-1-Mito treatment (MitoB2 and Mito $\rho^{\circ}$ ) or without treatment. b-d The time course for mtDNA copy number (b) and mitochondrial content (mitomass; $\mathbf{c}, \mathbf{d}$ ) was determined at different time points after treatment. Replication of mtDNA was determined by quantifying the mtDNA ND1 gene copies relative to the invariable $\beta$-globin nuclear gene (b). Mito- chondrial content was determined by flow cytometry using $10-n-$ nonyl acridine orange (NAO) dye (c, d). e, f Mitochondrial dynamics-related proteins (fusion proteins OPA1 and MFN2, fission proteins Fis1 and Drp1) were analysed (e) and quantified (f) by Western blotting after 2 days of treatment. Parent cybrid cells (C2) without mitochondrial delivery were used as the control. ${ }^{a} \mathrm{p}<0.05$ : significant difference compared with the $\mathrm{C} 2$ group or mock control group $\left(\mathrm{B} 2\right.$ and $\left.\rho^{\circ}\right) ;{ }^{\mathrm{b}} \mathrm{p}<0.05$ : significant difference between $\mathrm{B} 2$ and $\rho^{\circ}$ groups; ${ }^{\mathrm{c}} \mathrm{p}<0.05$ : significant difference between MitoB2 and Mito $\rho^{\circ}$ groups $(\mathrm{n}=3)$.

(online suppl. fig. 1a), mtDNA replication (online suppl. fig. $1 b$ ) and mitochondrial dynamic proteins (online suppl. fig. 1c) were not affected by Pep-1 alone in cells before and after 2 days of treatment.

\section{Loading Capacity of Mitochondrial Calcium in Rescued Cells}

The $\mathrm{Ca}^{2+}$ indicator fluorophore rhod- 2 was used to monitor $\left[\mathrm{Ca}^{2+}\right] \mathrm{m}$ uptake, a factor induced by mitochondrial swelling and depolarization via a 2 -step cold loading/warm incubation protocol, which achieved exclusive loading of rhod-2 into mitochondria (fig. 6). After labelling cellular mitochondria with MitoTracker Green dye, a marked increase in mitochondrial rhod-2 fluorescence was observed by confocal microscopy in MERRF cybrid 


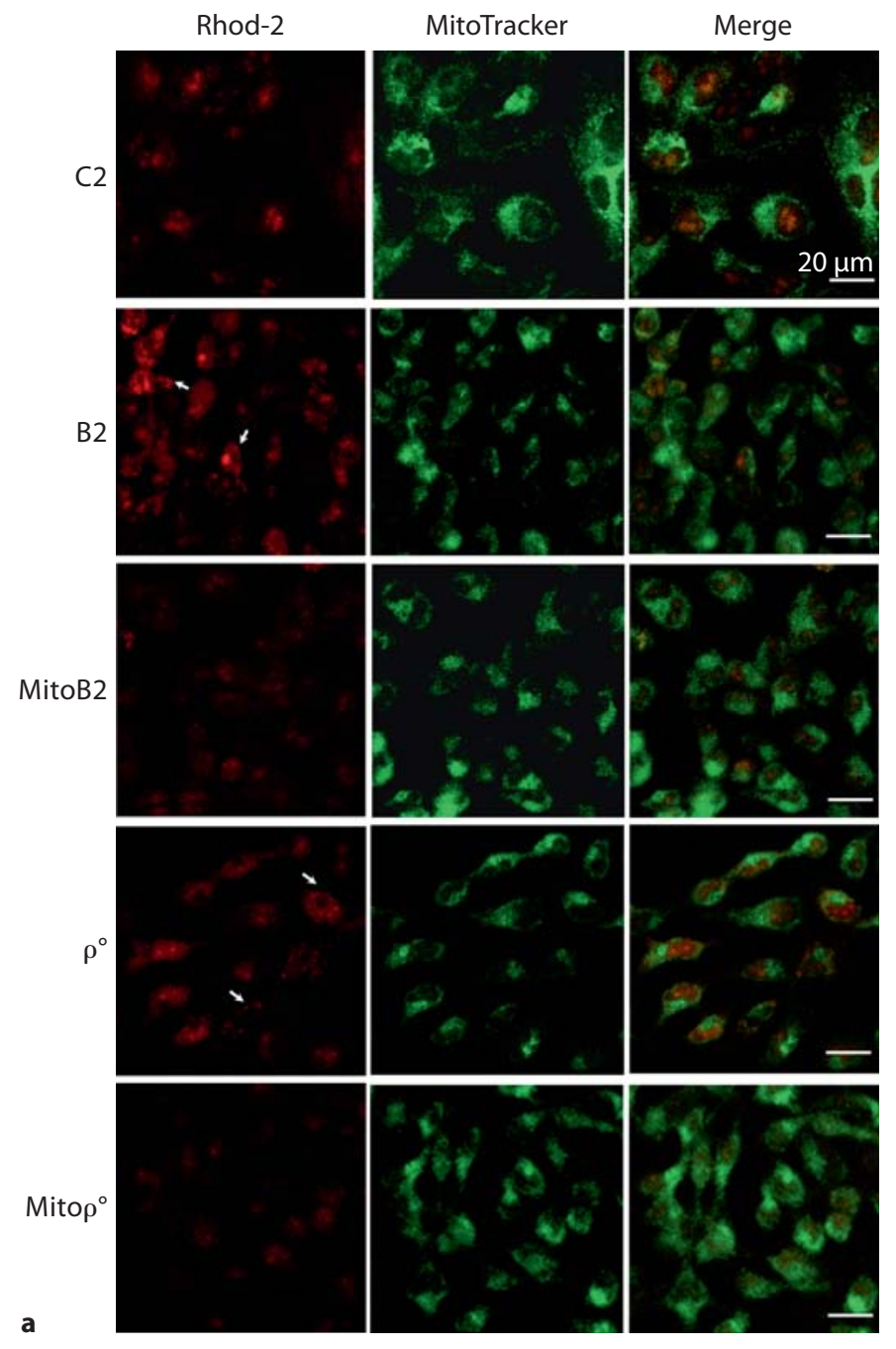

a

(MitoB2) and $\rho^{\circ}\left(\right.$ Mito $\left.^{\circ}\right)$ cells. In contrast, both cell types exhibited decreased expression after 1 day of PMD treatment (fig. 6a, arrows). To quantify the loading capacity of mitochondrial calcium with or without $\mathrm{H}_{2} \mathrm{O}_{2}$, rhod-2loaded cells were immediately analysed before (baseline) and after additional treatment with $\mathrm{H}_{2} \mathrm{O}_{2}(0.15 \%$ concentration in $\mathrm{PBS}, \mathrm{w} / \mathrm{v}$; fig. $6 \mathrm{~b})$. These results consistently showed that PMD treatment significantly diminished the loading capacity of mitochondrial calcium in MERRF cybrid and $\rho^{\circ}$ cells (fig. 6 b; $p<0.05$ ). Influx of mitochondrial calcium dramatically increased in cells affected by oxidative stress induced by $\mathrm{H}_{2} \mathrm{O}_{2}$ but was still lower in PMD-treated MERRF cybrid and $\rho^{\circ}$ cells than in each of the untreated groups (fig. $6 \mathrm{~b} ; \mathrm{p}<0.05$ ).

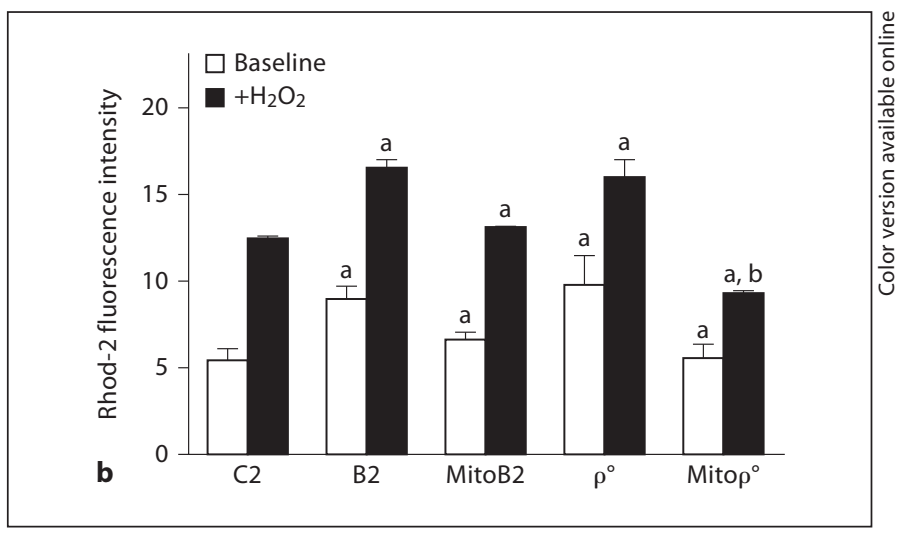

Fig. 6. Mitochondrial calcium uptake capacity in cells after mitochondrial delivery. a Mitochondrial calcium uptake was analysed by a cold loading/warm protocol that provided exclusive loading of the fluorophore rhod-2-AM into the mitochondria in MERRF cybrid (B2) and $\rho^{\circ}$ cells with 2 days of Pep-1-Mito mitochondria treatment (MitoB2 and Mito $\rho^{\circ}$ ) or without treatment. Mitochondria in treated cells were co-stained with MitoTracker Green (green) and used to visualize calcium uptake in mitochondria, as determined by co-localization with rhod-2 red fluorescence. As indicated by the arrows, marked, extensive fluorescence of mitochondrial calcium was found in the $\mathrm{B} 2$ and $\rho^{\circ}$ groups. $\mathbf{b}$ To further quantify the loading of mitochondrial calcium, the fluorescence intensity of rhod-2 in treated cells was immediately determined by flow cytometry (baseline expression). Each sample was additionally treated with $0.15 \% \mathrm{H}_{2} \mathrm{O}_{2}$ to induce calcium overloading into mitochondria. After $40 \mathrm{~min}$ of stimulation, fluorescence intensity was analysed again for the same conditions. Parent cybrid cells (C2) without mitochondrial delivery were used as the control. ${ }^{a} \mathrm{p}<0.05$ : significant difference compared with the $\mathrm{C} 2$ group or mock control group (B2 and $\left.\rho^{\circ}\right) ;{ }^{b} \mathrm{p}<0.05$ : significant difference between MitoB2 and Mito $\rho^{\circ}$ groups $(n=3)$.

\section{Discussion}

Spees et al. [8] showed that the respiration of $\rho^{\circ}$ cells could be rescued in vitro via mitotransfer and did not result from either cell fusion or a passive internalization process. It is unclear whether whole functional mitochondria or mtDNA was transferred, and if it was the former, then whether cytoplasmic transfer was required. Here, we used a novel approach of active intervention to manipulate mitotransfer by utilizing PMD and demonstrated that functional recovery of deficient mitochondria in a cybrid model required neither cell-cell contact nor cytoplasmic transfer, consistent with the findings of another study [10].

Our results show that Pep-1-labelled mitochondria can not only be successfully internalized with differ- 
ent delivery efficiencies depending on the cell type, but also continue to proliferate for up to 15 days in rescued MERRF cybrid and $\rho^{\circ}$ cells. This was shown by increased GFP expression in these cells following delivery of mitochondria-tagged GFP. Cybrid cells, which are widely used to study neurodegenerative diseases [23], are suitable for studying genotype-phenotype relationships in cell culture and truly reflect the phenotype caused by mtDNA alterations without nuclear genome interference [20]. The regulatory effects of foreign mitochondria on mitochondrial homeostasis of host cells were also investigated by comparing different models of mitochondrial injury, namely a point mutation in MERRF cybrid cells and ethidium bromide-induced mtDNA depletion in $\rho^{\circ}$ cells.

In the present study, PMD consistently recovered mitochondrial integrity in both types of treated cells as demonstrated by the increase in mitochondrial biogenesis; however, PMD differentially affected the expression of mitochondrial dynamics-related proteins. Inhibition of mitochondrial fission and promotion of mitochondrial fusion were observed in rescued MERRF cybrid cells and $\rho^{\circ}$ cells, respectively. In general, fusing damaged mitochondria in normal cells with neighbouring, intact mitochondria restores the functionality of damaged mitochondria $[24,25]$. However, PMD did not improve mitochondrial fusion in MERRF cybrid cells, suggesting that it could be associated with the incomplete function of the endogenous mitochondria caused by the mtDNA mutation in MERRF cybrid cells. Thus, the properties of endogenous mitochondria in target cells could be a factor that affects the regulatory pathways and outcomes of mitochondrial therapy via PMD.

In addition to its observed effects on mitochondrial biogenesis, PMD also caused a drastic downregulation of the MFN2 protein, but not of the OPA1 protein, in rescued MERRF cybrid cells. MFN2, a dynamin-like GTPase, and OPA1 are regulators of mitochondrial dynamics. However, MFN2 regulates mitochondrial metabolism independently of its fusion function [26] and has several extra-mitochondrial functions and signalling roles, such as modulating $\left[\mathrm{Ca}^{2+}\right] \mathrm{m}$ uptake [26]. MFN2 is enriched in the mitochondrion-associated membrane tethered to the endoplasmic reticulum (ER) [26] and controls the efficiency of $\left[\mathrm{Ca}^{2+}\right] \mathrm{m}$ uptake by determining the inter-organelle distance [27]. In the present study, we not only demonstrated the co-localization of delivered mitochondria and endogenous mitochondria in host cells but also showed that the overloading of $\left[\mathrm{Ca}^{2+}\right] \mathrm{m}$ with or without inducing oxidative stress was dramatically reduced in both types of treated cells. These findings suggest that

Mitochondrial Therapy for MERRF Syndrome steric hindrance resulting from the translocation of foreign mitochondria interfered with the endogenous MFN2-ER connection and led to a reduction in the excessive uptake of $\left[\mathrm{Ca}^{2+}\right] \mathrm{m}$, which in turn triggered mitochondrial permeability transition pore opening and cell death [28]. Recently, an ER-mitochondrial disorder was considered to be involved in the pathogenesis of Alzheimer's disease $[29,30]$, although it remains unidentified in other neurodegenerative diseases.

Based on our findings, we propose that blocking the original MFN2-ER connection may aid in treating MERRF syndrome via modulating ER-mitochondrial calcium signalling. Resetting calcium overloading by treatment with mitochondrial calcium channel blockers in cells derived from MERRF and Leigh syndrome patients can ameliorate disease progression $[31,32]$ and is a potential therapeutic strategy for mitochondrial diseases [4]. We also observed changes in cell morphology, with lamellipodial protrusions surrounding MERRF cybrid cells immediately after PMD treatment. This finding supports the idea that cytoskeleton network suppression is another pathogenic factor in MERRF fibroblasts [33]. Activation of lamellipodia formation has been implicated in promoting mitochondrial trafficking and functions [34-36]. Moreover, mitochondrial movement is regulated by mitochondrial Rho GTPase (Miro), which acts as a $\mathrm{Ca}^{2+}$-dependent adaptor between mitochondria and the cytoskeleton. High levels of $\mathrm{Ca}^{2+}$ discharged from the ER upon binding to Miro cause a conformational change in Miro, which allows it to sequester the motor heads of kinesin heavy chains and inhibit microtubule-driven movements $[37,38]$. Here, activating the cytoskeleton via PMD consistently showed $\left[\mathrm{Ca}^{2+}\right] \mathrm{m}$ variations in MERRF cybrid cells.

In our cybrid model of PMD, the efficiency of mitochondrial delivery was dependent on different carrier/ cargo ratios (data not shown) because the size and homogeneity of nanoparticles determined the efficiency of cell entry $[11,39]$. In our previous study [15] and another study [14], the optimal delivery efficiency of quantum dot nanoparticles via Pep-1 was at a concentration ratio of 6,000:1 (Pep-1 to quantum dot nanoparticles). Here, we found a better weight ratio of 1:1,750 than 1:5,250 and 1:1,050 with a higher fluorescent labelling intensity of mitochondria detected by flow cytometry. However, noninternalized mitochondria were still observed floating in the culture medium $48 \mathrm{~h}$ after delivery (data not shown). This may have been due to mitochondrial sizes that can range from 500 to $1,000 \mathrm{~nm}$, which are too large for Pep1 delivery. 
A Pep-1-cargo complex is formed non-covalently as with nanoparticles, and the complex size determines the appropriate peptide concentration, which varies depending on the cargo. Larger complexes produced by aggregation are formed by an increased amount of peptide [39]. Complexes larger than approximately $800 \mathrm{~nm}$ in diameter and smaller than $200 \mathrm{~nm}$ in diameter result in poor biological responses [11, 39]. Because of the incomplete internalization of Pep-1-labelled mitochondria in this system, the effective threshold amount of internalized mitochondria could not be exactly determined. According to a previous study [8], 100 cells can sufficiently rescue 1 cell clone via mitotransfer. Here, we found that the range of mitochondrial treatment with doses of $>1,050$ $\mathrm{ng} /$ per cell or $<210 \mathrm{ng} /$ per cell $(525 \mathrm{ng} /$ per cell was effective in this study) was ineffective in restoring $\Delta \psi \mathrm{m}$ in MERRF cybrid cells under PMD treatment for 2 days (data not shown).

A perceived improvement in mitochondrial function is not always related to an obvious decrease in the proportion of mutant mtDNA, which makes it difficult to assess a change in mtDNA heteroplasmy [40]. Because an obvious decrease in the mtDNA mutation load in the rescued MERRF cybrid cells was not observed in our study, it was necessary to evaluate the mitochondrial homeostasis regulatory mechanisms. It was noted that PMD significantly upregulated the expression of biogenesis-related genes including NRF-1 and Tfam and increased mtDNA replication in both of the cell types that were treated. In addition, this effect was not caused by treatment with Pep-1 alone (online suppl. results). Stimulation of biogenesis signalling is one of the candidate therapeutic approaches for treating mitochondrial diseases, but it is not a reasonable indicator of treatment efficacy. An increase in mitochondrial proliferation usually occurs with all mitochon- dria, irrespective of whether they are dysfunctional [4, 41]. This explains why mitochondrial hyperproliferation in muscle (ragged-red fibres) is often found in patients with pathogenic mtDNA mutations [42]. Recently, analysis of mitochondrial dynamics appeared to reflect mitochondrial homeostasis.

Alterations in mitochondrial dynamics have been implicated in neurodegenerative diseases, such as Parkinson's disease [43], amyotrophic lateral sclerosis [44] and Alzheimer's disease [30], although their role in MERRF syndrome remains unclear. Based on our findings, we propose that preventing further mitochondrial damage and inhibiting mitochondrial fission is a priority for developing a therapeutic strategy for the treatment of MERRF syndrome. Furthermore, we have also demonstrated the therapeutic value of PMD in MERRF skin fibroblasts, as evidenced by an approximately twofold increase in $\Delta \psi \mathrm{m}$ compared with cells without treatment (data not shown). However, further investigation and confirmation of whether PMD can rescue cells in the long term and whether it is a suitable therapy for other mitochondrial disorders are required.

\section{Acknowledgments}

This study was supported by research grants from Changhua Christian Hospital (99-ICO-01) and the National Science Council (NSC 100-2314-B-371-003). We gratefully appreciate the kind assistance of Mr. Shi-Bei Wu in the supply of cybrids and $\rho^{\circ}$ cells from a patient with MERRF syndrome established in the laboratory of Prof. Y.H. Wei., Prof. H.L. Su for device support with the confocal microscope in the Center of Tissue Engineering and Stem Cells Research (National Chung Hsing University, Taiwan) and Prof. S.F. Weng (Institute of Molecular Biology, National Chung Hsing University, Taiwan) for support with genetic recombination techniques.

\section{References}

1 Shoffner JM, Lott MT, Lezza A, Seibel P, Ballinger SW, Wallace DC: Myoclonic epilepsy and ragged-red fiber disease (MERRF) is associated with a mitochondrial DNA tRNA ${ }^{\text {Lys }}$ mutation. Cell 1990;61:931-937.

$\checkmark 2$ Luft R: The development of mitochondrial medicine. Proc Natl Acad Sci USA 1994;91: 8731-8738.

3 Chan DC: Mitochondria: dynamic organelles in disease, aging, and development. Cell 2006;125:1241-1252.

-4 Schon EA, DiMauro S, Hirano M, Gilkerson RW: Therapeutic prospects for mitochondrial disease. Trends Mol Med 2010;16:268-276.
5 Kagawa Y, Inoki Y, Endo H: Gene therapy by mitochondrial transfer. Adv Drug Deliv Rev 2001;49:107-119.

6 King MP, Attardi G: Injection of mitochondria into human cells leads to a rapid replacement of the endogenous mitochondrial DNA. Cell 1988;52:811-819.

7 Tachibana M, Sparman M, Sritanaudomchai H, Ma H, Clepper L, Woodward J, Li Y, Ramsey C, Kolotushkina O, Mitalipov S: Mitochondrial gene replacement in primate offspring and embryonic stem cells. Nature 2009;461:367-372.
8 Spees JL, Olson SD, Whitney MJ, Prockop DJ: Mitochondrial transfer between cells can rescue aerobic respiration. Proc Natl Acad Sci USA 2006;103:1283-1288.

$\checkmark$ McCully JD, Cowan DB, Pacak CA, Toumpoulis IK, Dayalan H, Levitsky S: Injection of isolated mitochondria during early reperfusion for cardioprotection. Am J Physiol Heart Circ Physiol 2009;296:H94H105.

10 Pinkert CA, Irwin MH, Johnson LW, Moffatt RJ: Mitochondria transfer into mouse ova by microinjection. Transgenic Res 1997;6:379383. 
-11 Morris M, Deshayes S, Heitz F, Divita G: Cell-penetrating peptides: from molecular mechanisms to therapeutics. Biol Cell 2008; 100:201-217.

$\checkmark 12$ Lundberg P, Langel U: A brief introduction to cell-penetrating peptides. J Mol Recognit 2003; 16:227-233.

13 Gros E, Deshayes S, Morris MC, AldrianHerrada G, Depollier J, Heitz F, Divita G: A non-covalent peptide-based strategy for protein and peptide nucleic acid transduction. Biochim Biophys Acta 2006;1758:384-393.

14 Mattheakis LC, Dias JM, Choi YJ, Gong J, Bruchez MP, Liu J, Wang E: Optical coding of mammalian cells using semiconductor quantum dots. Anal Biochem 2004;327:200208.

15 Chang JC, Su HL, Hsu S: The use of peptidedelivery to protect human adipose-derived adult stem cells from damage caused by the internalization of quantum dots. Biomaterials 2008;29:925-936.

16 Morris MC, Depollier J, Mery J, Heitz F, Divita G: A peptide carrier for the delivery of biologically active proteins into mammalian cells. Nat Biotechnol 2001;19:1173-1176.

-17 Mattheakis LC, Dias JM, Choi YJ, Gong J, Bruchez MP, Liu J, Wang E: Optical coding of mammalian cells using semiconductor quantum dots. Anal Biochem 2004;327:200208.

18 Moschos S, Williams A, Lindsay M: Cellpenetrating-peptide-mediated siRNA lung delivery. Biochem Soc Trans 2007;35:807810 .

19 Liu CY, Lee CF, Hong CH, Wei YH: Mitochondrial DNA mutation and depletion increase the susceptibility of human cells to apoptosis. Ann NY Acad Sci 2004;1011:133-145.

20 King MP, Attardi G: Human cells lacking mtDNA: repopulation with exogenous mitochondria by complementation. Science 1989; 246:500-503.

21 Brooks C, Wei Q, Feng L, Dong G, Tao Y, Mei L, Xie ZJ, Dong Z: Bak regulates mitochondrial morphology and pathology during apoptosis by interacting with mitofusins. Proc Natl Acad Sci USA 2007;104:1164911654.

-22 Trollinger DR, Cascio WE, Lemasters JJ: Selective loading of Rhod 2 into mitochondria shows mitochondrial $\mathrm{Ca} 2+$ transients during the contractile cycle in adult rabbit cardiac myocytes. Biochem Biophys Res Commun 1997;236:738-742.
23 Beal MF: New techniques for investigating mitochondrial DNA in neurodegenerative diseases. Neurology 1997;49:907-908.

24 Detmer SA, Chan DC: Functions and dysfunctions of mitochondrial dynamics. Nat Rev Mol Cell Biol 2007;8:870-879.

25 Mouli PK, Twig G, Shirihai OS: Frequency and selectivity of mitochondrial fusion are key to its quality maintenance function. Biophys J 2009;96:3509-3518.

26 De Brito OM, Scorrano L: Mitofusin 2 tethers endoplasmic reticulum to mitochondria. Nature 2008;456:605-610.

27 Arnaudeau S, Kelley WL, Walsh JV, Demaurex N: Mitochondria recycle $\mathrm{Ca} 2+$ to the endoplasmic reticulum and prevent the depletion of neighboring endoplasmic reticulum regions. J Biol Chem 2001;276:29430-29439.

28 Jou MJ, Peng TI, Hsu LF, Jou SB, Reiter RJ, Yang CM, Chiao CC, Lin YF, Chen CC: Visualization of melatonin's multiple mitochondrial levels of protection against mitochondrial $\mathrm{Ca} 2+$ mediated permeability transition and beyond in rat brain astrocytes. J Pineal Res 2010;48:20-38.

29 Schon EA, Area-Gomez E: Is Alzheimer's disease a disorder of mitochondria-associated membranes? J Alzheimers Dis 2010;20: 281-292.

30 Area-Gomez E, de Groof AJC, Boldogh I, Bird TD, Gibson GE, Koehler CM, Yu WH, Duff KE, Yaffe MP, Pon LA: Presenilins are enriched in endoplasmic reticulum membranes associated with mitochondria. Am J Pathol 2009;175:1810-1816.

- 31 Brini M, Pinton P, King MP, Davidson M, Schon EA, Rizzuto R: A calcium signaling defect in the pathogenesis of a mitochondrial DNA inherited oxidative phosphorylation deficiency. Nat Med 1999;5:951-954

32 Visch HJ, Rutter GA, Koopman WJH, Koenderink JB, Verkaart S, de Groot T, Varadi A, Mitchell KJ, van den Heuvel LP, Smeitink JAM: Inhibition of mitochondrial $\mathrm{Na}+$ $\mathrm{Ca} 2+$ exchange restores agonist-induced ATP production and $\mathrm{Ca} 2+$ handling in human complex I deficiency. J Biol Chem 2004; 279:40328-40336.
33 Ma YS, Chen YC, Lu CY, Liu CY, Wei YH: Upregulation of matrix metalloproteinase 1 and disruption of mitochondrial network in skin fibroblasts of patients with MERRF syndrome. Ann NY Acad Sci 2005;1042:55-63.

34 Mogilner A, Oster G: Cell motility driven by actin polymerization. Biophys J 1996;71: 3030-3045.

35 Rappaport L, Oliviero P, Samuel JL: Cytoskeleton and mitochondrial morphology and function. Mol Cell Biochem 1998;184: 101-105.

36 Rusanen H, Annunen J, Yla Outinen H, Laurila A, Peltonen J, Hassinen IE, Majamaa K: Cytoskeletal structure of myoblasts with the mitochondrial DNA 3243A->G mutation and of osteosarcoma cells with respiratory chain deficiency. Cell Motil Cytoskeleton 2002;53:231-238.

-37 Kornmann B, Walter P: ERMES-mediated ER-mitochondria contacts: molecular hubs for the regulation of mitochondrial biology. J Cell Sci 2010;123:1389-1393.

38 Wang X, Schwarz TL: The mechanism of $\mathrm{Ca} 2+$-dependent regulation of kinesin-mediated mitochondrial motility. Cell 2009; 136:163-174.

39 Munoz-Morris MA, Heitz F, Divita G, Morris MC: The peptide carrier Pep-1 forms biologically efficient nanoparticle complexes. Biochem Biophys Res Commun 2007;355: 877-882.

40 Ahola-ErkkilaS, CarrollCJ, Peltola-Mjosund K, Tulkki V, Mattila I, Seppanen-Laakso T, Oresic M, Tyynismaa H, Suomalainen A: Ketogenic diet slows down mitochondrial myopathy progression in mice. Hum Mol Genet 2010;19:1974-1984.

41 Scarpulla RC: Transcriptional paradigms in mammalian mitochondrial biogenesis and function. Physiol Rev 2008;88:611-638.

42 DiMauro S, Schon EA: Mitochondrial respiratory-chain diseases. N Engl J Med 2003; 348:2656-2668.

43 Narendra D, Tanaka A, Suen DF, Youle RJ: Parkin is recruited selectively to impaired mitochondria and promotes their autophagy. J Cell Biol 2008;183:795-803.

-44 Magrane J, Manfredi G: Mitochondrial function, morphology, and axonal transport in amyotrophic lateral sclerosis. Antioxid Redox Signal 2009;11:1615-1626.
Mitochondrial Therapy for MERRF Syndrome 